\title{
Mitigating Spectral Leakage and Sampling Errors in Spatial and Spectral $\left(S^{2}\right)$ Imaging
}

\author{
D. R. Gray*, S. R. Sandoghchi, N. V. Wheeler, N.K. Baddela, G. T. Jasion, M. N. Petrovich, F. Poletti and \\ D. J. Richardson \\ Optoelectronics Research Centre, University of Southampton, Southampton, SO171BJ, UK, \\ *D.R.Gray@soton.ac.uk
}

\begin{abstract}
We present a novel method for validating the relative power value (MPI) of the Spatial and Spectral $\left(\mathrm{S}^{2}\right)$ imaging technique. By applying corrections for spectral leakage and sampling errors we found the MPI determinations to be accurate within $1 \mathrm{~dB}$.

OCIS codes: (060.2270) Fiber characterization; (060.4005) Microstructured fibers
\end{abstract}

\section{Introduction}

Multimode fibers are a topic of major interest for applications in telecommunications (Spatial Division Multiplexing), high-power fiber lasers/amplifiers and laser power delivery. Spatial and Spectral $\left(\mathrm{S}^{2}\right)$ imaging, originally developed by Nicholson et al. [1] yields a host of information with no prior knowledge about the modal structure of the fiber under test (FUT) required and can detect very small fractions of power present in higher order modes, and has thus quickly established itself as the most widely used technique to analyze the modal content of multimode fibers. $S^{2}$ provides the relative modal power, or Multipath Interference (MPI), Differential Group Delay (DGD) and phase difference of the Higher Order Modes (HOM) relative to the dominant mode (typically the fundamental mode (FM)), as well as the spatial power distribution of all the optical modes it detects. Since its first demonstration, a number of improvements of the $S^{2}$ technique have been demonstrated, aimed at making it more versatile, sensitive and suited for a variety of characterization problems. Recently, efforts have been directed to achieving real-time operation for the $S^{2}$ technique [2]. This was accomplished by modifying an existing method, based on a tunable laser source (TLS) and CCD camera [3] (Fig. 1); here, the TLS is continuously wavelength scanned over the measurement bandwidth whilst providing a triggering signal which enables the CCD to capture the output intensity distribution of the FUT at much faster rates than was previously possible [2].

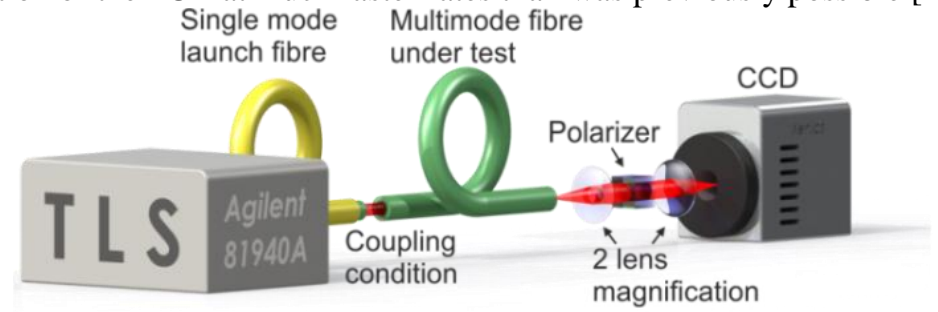

Fig. 1. Diagram of a TLS \& CCD based $\mathrm{S}^{2}$ imaging system.

On the other hand, more improvements are required, for instance, in order to fully establish the accuracy of the technique in relation to MPI determination and how this is affected by the choice of measurement parameters and/or by the procedure used for the data analysis. In this paper we identify two potential sources of substantial error, namely sampling and spectral leakage, quantify their impact and identify strategies to minimize them. We also devise a straightforward method to accurately validate the measured MPI values. Finally, we apply the findings to the analysis of a novel 19 cell HC-PBGF and quantify the impact of the corrections identified.

\section{Sampling Error and Spectral Leakage}

$\mathrm{S}^{2}$ measures the intensity modulation due to modal interference (MI) at each pixel as a function of the wavelength. This is converted from wavelength to frequency and then to time via Fast Fourier Transformed (FFT) to produce an MPI vs. DGD plot [1]. In this procedure, however, spectral leakage (SL), a well-known effect [4], results when the measurement bandwidth is not an exact multiple of the period of the MI modulation and causes the peak MPI value to be reduced by a quantity ranging from 0 to $\sim 3.9 \mathrm{~dB}$ [4]. Additionally, when the MI signal is discretized in wavelength and then converted into frequency, a sampling error may occur as a consequence of the peaks and troughs of the MI modulation being inaccurately captured by the discretization process. The sampling error produces an MPI value that is generally lower than its true value. We accurately modeled these errors by generating a "reference" $S^{2}$ signal corresponding to an idealized fiber having a single HOM with fixed MPI and a known DGD. 
By varying the DGD we were able to investigate the trends in the amplitude of SL and sampling errors, see Fig. 2(a). The blue points show the difference between calculated MPI values and the theoretical value (set at $-23 \mathrm{~dB}$ in this case). The sampling error, shown by the green symbols, was found to increase monotonically as the DGD value approaches the Nyquist sampling frequency, with the maximum MPI error depending on the bandwidth and on the resolution of the measurement. The SL error depends on the particular value of DGD, and as seen in the plot it oscillates between 0 and $\sim 3.9 \mathrm{~dB}$, the maximum theoretical value. The red symbols show a worst-case scenario with sampling and maximum SL errors combined. We found that the maximum SL error can be reduced from $\sim 3.9 \mathrm{~dB}$ to $\sim 0.3 \mathrm{~dB}$ (as shown in Fig. 2(b)) by computing the MPI as sum of 3 points at either side of the experimental peak value. The sampling error can be minimized by suitably reducing the measured bandwidth, Fig. 2(b). We found that, for 2-3 nm bandwidth at $10 \mathrm{pm}$ resolution, the error can be kept to $<1 \mathrm{~dB}$, but if wider measurement bandwidths are employed, the error can be as high as several dB.
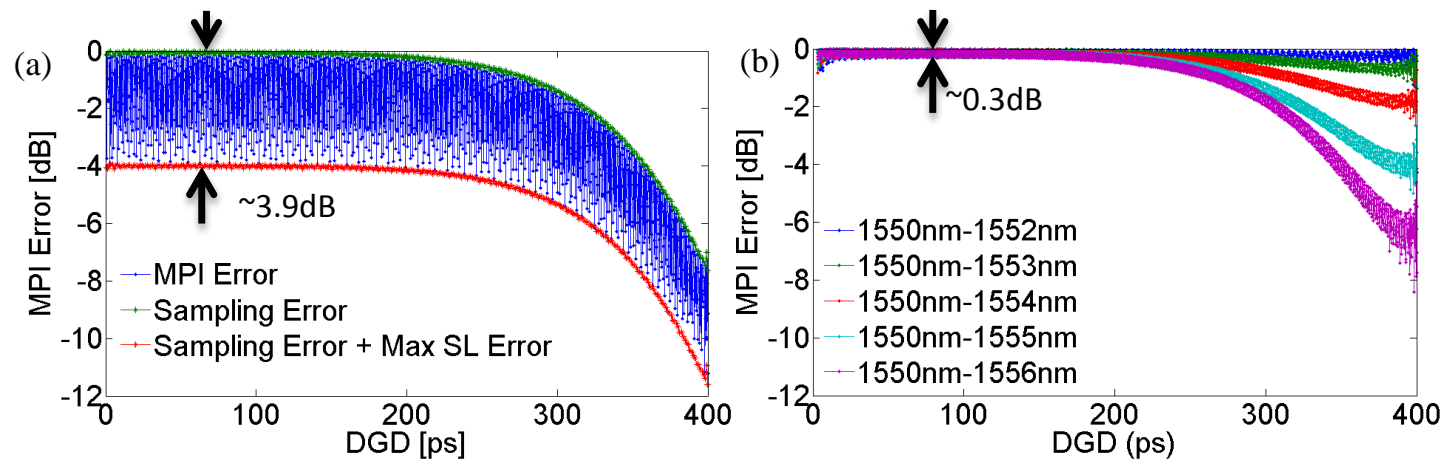

Fig. 2: (a) Analysis of spectral leakage and sampling errors vs. DGD for a given set of experimental conditions (1550-1557 nm bandwidth, 10pm resolution), showing the sampling error increasing with DGD and the SL error oscillating between 0 and $\sim 3.9 \mathrm{~dB}$. (b) Strategies to minimize errors: SL is reduced to $\sim 0.3 \mathrm{~dB}$ by estimating the MPI as sum of 3 points at either side of the peak value; the sampling error can be reduced to $<1$ $\mathrm{dB}$ by reducing the measurement bandwidth (to $\leq 3 \mathrm{~nm}$ in this particular instance).

\section{Theoretical Validation of MPI value}

A validation of MPI values determined via $S^{2}$ analysis has been previously obtained by using a long period grating [1]. Here we follow a simpler approach that does not require complex grating fabrication: we use Fresnel reflection from a suitable optical element (a sapphire window), interposed in the measurement beam path, to create a "virtual" HOM through a delayed copy of the FM beam, Fig. 3(a). The MPI and DGD of the virtual HOM can be related to the optical properties of the window via the following equations:

$$
M P I=10 \log _{10}\left(R^{2}\right) ; \quad D G D=\frac{2 t n-2 t n^{2} \sin ^{2} \theta}{c \cos \theta}
$$

where $\mathrm{R}$ is the Fresnel reflectance, $n(\lambda)$ the wavelength dependent refractive index, $t$ the thickness of the sapphire window and $\theta$ is the beam propagation angle from Snell's law.
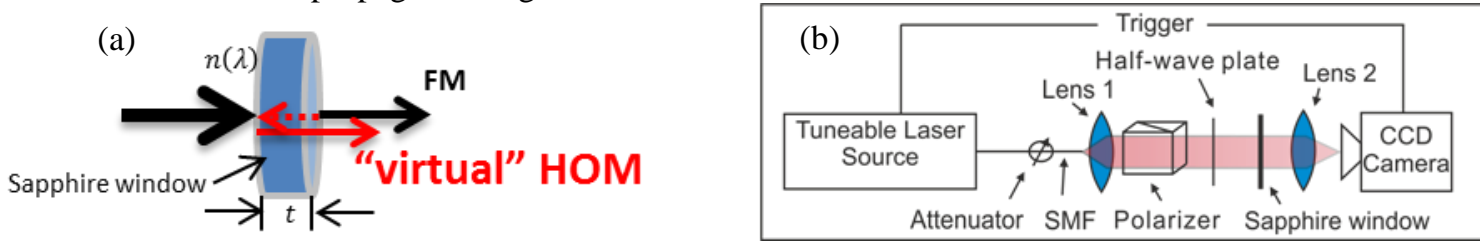

Fig. 3: (a) Virtual HOM method for MPI validation; (b) Schematic of the modified $\mathrm{S}^{2}$ setup.

We used a $4 \mathrm{~mm}$ thick sapphire window (n 1.77) interposed between the polarizing optics and the CCD in the collimated output beam from a single mode fiber, Fig. 3(b). We collected angle resolved measurements as the sapphire window was tilted from $-20^{\circ}$ to $20^{\circ}$ at $1^{\circ}$ intervals; this modifies the reflected beam path and thus the DGD of the virtual HOM. Measurements were taken in the range 1550-1554 nm at $10 \mathrm{pm}$ resolution. We first investigated the parallel polarization incident on the window. As shown in Fig. 4(a), the uncorrected peak MPI value is as expected systematically lower than the theoretical value from Eq. 1(a), and the angular dependence is a consequence of SL error dependence on DGD of the virtual HOM (which is a function of $\theta$, Eq. 1(b)). By minimizing the SL error as detailed in the previous section, excellent agreement was found with the exact MPI value. Fig. 4(b) shows a summary of results for the two incident polarizations, demonstrating a $<1 \mathrm{~dB}$ MPI error in both cases. 

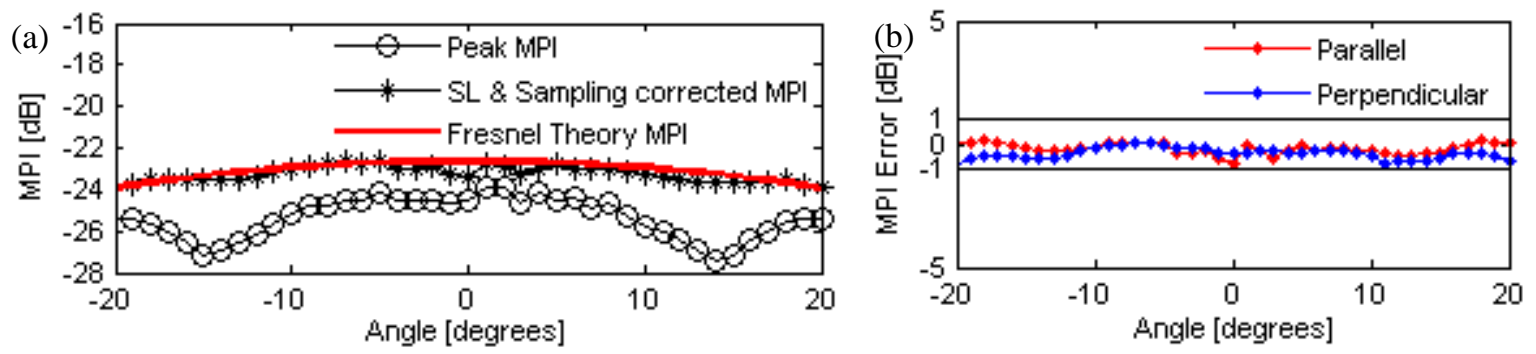

Fig. 4: (a) Comparison between theoretical and experimental MPI values for a "virtual" HOM generated by the sapphire window, as a function of angle of incidence; (b) Residual MPI error (both polarizations) after SL and sampling error corrections implemented.

\section{Measurement of a HC-PBGF}

We investigated the effect of SL and sampling errors on the $S^{2}$ measurement of a 19 cell hollow core photonic band gap fiber (HC-PBGF). The fiber has a minimum loss of $3.5 \mathrm{~dB} / \mathrm{km}$ at $\sim 1500 \mathrm{~nm}$ and a very wide, surface mode free, central region of the bandgap with a 3-dB transmission bandwidth of $160 \mathrm{~nm}$ [5]. The $\mathrm{S}^{2}$ results are plotted in Fig. 5(a), showing the peaks and intensity distributions corresponding to the main optical modes of the HC-PBGF. We estimate the errors due to sampling and SL as detailed in Sec. 2, see Fig. 5(b). The error due to SL is found to oscillate between 0 and $\sim 4 \mathrm{~dB}$, in good agreement with theory, and similarly the sampling error is found to increase with increasing DGD. If these effects were not accounted for, the MPI value could be significantly underestimated by between $1.48 \mathrm{~dB}$ to $9.3 \mathrm{~dB}$ for this measurement.

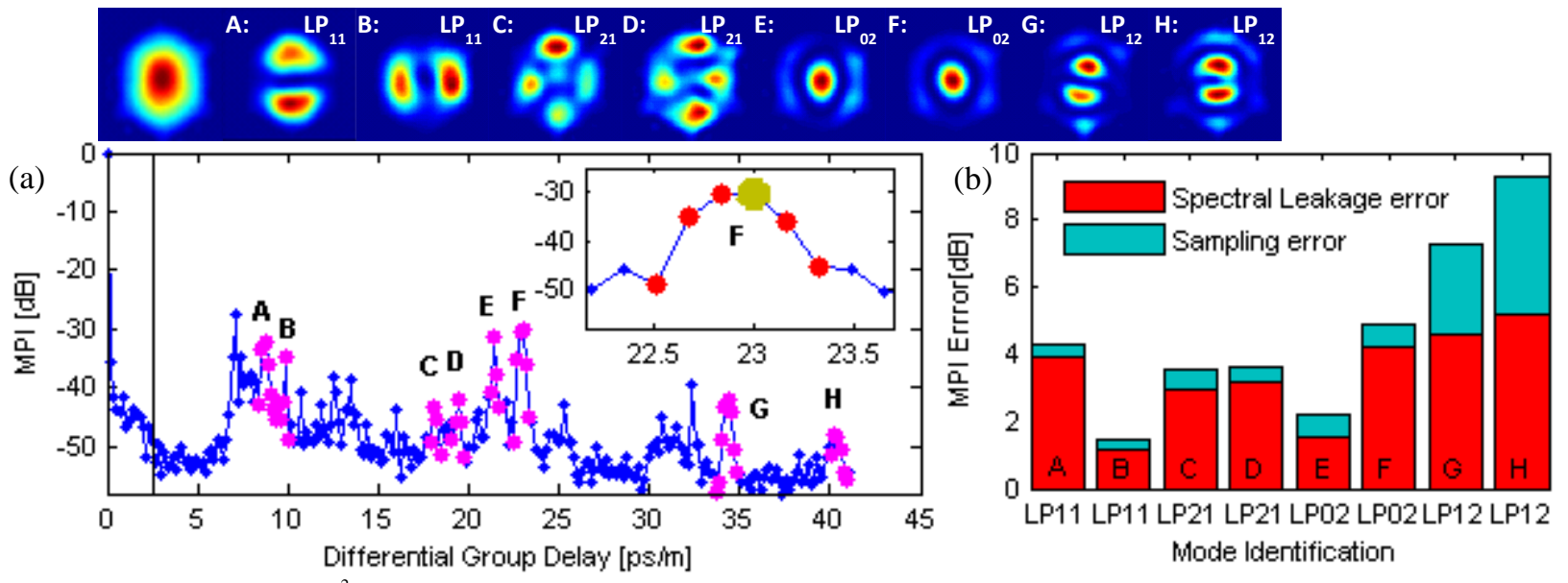

Fig. 5: (a) $\mathrm{S}^{2}$ of a 19c HC-PBGF with a selection. (b) SL and sampling errors corresponding to each peak.

\section{Conclusion}

We have performed a thorough analysis of the accuracy of MPI determinations in a typical $\mathrm{S}^{2}$ measurement. We have identified sampling and spectral leakage as potential sources of significant errors and have identified effective strategies to minimize their effect. We have also presented an experimentally simple method for accurately validating the relative power values (MPI). We have achieved this by creating a "virtual" HOM with known DGD and MPI through the use of a sapphire window. The impact of errors due to sampling and spectral leakage in the measurement of a 19 cell HC-PBGF has been evaluated.

This work was supported by the EU 7th Framework Programme under grant agreement 228033 (MODE-GAP).

\section{References}

[1] J. W. Nicholson, et. al., "Spatially and spectrally resolved imaging of modal content in large-mode-area fibers", Optics Express, Vol. 16, Issue 10 , pp. 7233-7243 (2008).

[2] D.R. Gray, et. al., "Towards Real-Time Mode Content Characterization of Multimode Fibers" in Proceedings of European Conference on Optical Communications, (Cannes, 2014), paper Th.1.4.3

[3] S. Blin et. al., "Simple Modal Analysis Method for Multi-Mode Fibers," in Proceedings of European Conference on Optical Communications,(Vienna, 2009), paper P1.16.

[4] National Instruments white paper, "The Fundamentals of FFT-Based Signal Analysis and Measurement in LabVIEW and LabWindows/CVI," http://www.ni.com/white-paper/4278/en/

[5] N. V. Wheeler, et. al. , "Wide-bandwidth, low-loss, 19-cell hollow core photonic band gap fiber and its potential for low latency data transmission," in Optical Fiber Communication Conference, (Optical Society of America, Los Angeles, 2012), paper PDP5A.2. 